\title{
Quantitative Evaluation of Dislocation Density in Epitaxial GaAs Layer on Si Using Transmission Electron Microscopy
}

\author{
Kangsik Kim, Jongyoung Lee, Hyojin Kim¹ , Zonghoon Lee* \\ School of Materials Science and Engineering, UIsan National Institute of Science and Technology (UNIST), Ulsan 689-798, Korea \\ ${ }^{1}$ Photonic-Bio Research Center, Korea Photonics Technology Institute (KOPTI), Gwangju 500-779, Korea
}

*Correspondence to:

Lee $\mathrm{Z}$,

Tel: +82-52-217-2327

Fax: +82-52-217-2309

E-mail: zhlee@unist.ac.kr

Received June 21, 2014

Revised June 23, 2014

Accepted June 23, 2014

\begin{abstract}
Dislocation density and distribution in epitaxial GaAs layer on Si are evaluated quantitatively and effectively using image processing of transmission electron microscopy image. In order to evaluate dislocation density and distribution, three methods are introduced based on line-intercept, line-length measurement and our coding with linescanning method. Our coding method based on line-scanning is used to detect the dislocations line-by-line effectively by sweeping a thin line with the width of one pixel. The proposed method has advances in the evaluation of dislocation density and distribution. Dislocations can be detected automatically and continuously by a sweeping line in the code. Variation of dislocation density in epitaxial GaAs films can be precisely analyzed along the growth direction on the film.
\end{abstract}

Key Words: Dislocation density, Dislocation distribution, Image processing, Transmission electron microscopy, GaAs

\section{INTRODUCTION}

The size of hetero epitaxial semiconductors is getting smaller, as electronic devices and components get smaller than ever (Moore, 1965). For small devices, defects such as dislocations and lattice mismatch in hetero epitaxial structures affect significantly to the overall performance of device. Especially a major defect, dislocations play a major role in the growth and properties of epitaxial layers (Wagner, 1998). Thus it is important to quantify dislocation distribution and evaluate dislocation density in epitaxial layers. Detailed dislocation density data where epitaxial layer is grown would provide dislocation effects onto the hetero epitaxial structures. A number of hetero epitaxial semiconductors are always sought for improving performance in the devices and high yield in production. Per this demand, quantification of dislocation density is critical for the development of future semiconductors.
In research on thin films, there are many tools are available for analyzing dislocation density including X-ray diffractometer, scanning electron microscopy, atomic force microscopy, and small-angle neutron scattering (Heuser, 1994). In contrast to other tools, transmission electron microscopy (TEM) provides highly accurate determination of dislocation dentistry based on direct observation and can clearly distinguish dislocations in thin films. However, the earlier quantification study of dislocation density by TEM was complicated and time consuming. In this study, we apply calculation methods to evaluate dislocation density, where dislocations might be self-annihilation of antiphase boundary in grown anti-phase domain on step surfaces of GaAs during the epitaxial growth. By using image processing on TEM images taken from epitaxial GaAs films, we suggests more efficient way to quantify dislocation density and distribution at the same time.

This work was supported by the Industrial Strategic technology development program (10041926, development of high density plasma technologies for thin film deposition of nanoscale semiconductor and flexible display processing) funded by the the Ministry of Science, ICT and Future Planning.

@ This is an open-access article distributed under the terms of the Creative Commons Attribution Non-Commercial License (http://creativecommons.org/licenses/by-nc/3.0) which permits unrestricted noncommercial use, distribution, and reproduction in any medium, provided the original work is properly cited.

Copyrights @ 2014 by Korean Society of Microscopy 


\section{MATERIALS AND METHODS}

\section{Materials}

In order to analyze dislocation density, we used GaAs epitaxial films on $2^{\circ}$-off tilted toward $<111>$ Si deposited by Metal Organic Chemical Vapor Deposition as an example. Because tilted Si forms step surfaces, GaAs on Si forms anti-phase domain boundary along the tilted orientation and that is known as the dislocation of GaAs. These anti-phase domain boundaries grown eventually tend to interact each other, and then the dislocations could disappear at the point of intersection and this is called self-annihilation (Kawabe \& Ueda, 1987).

\section{Methods}

The TEM specimen for cross sectional observation was prepared by tripod polishing and following Ar ion milling using PIPS (Gatan Inc., USA) for final thinning.

Analysis was performed by JEM-2100 (JEOL, Japan) with $\mathrm{LaB}_{6}$ gun operated at $200 \mathrm{kV}$ accelerating voltage. Several images were merged to observe the variation of dislocation density along the growth direction in larger area. Image J (National Institutes of Health, USA) and Photoshop ver. 8.0 (Adobe Systems Inc., USA) were used to distinguish dislocations from the TEM image, and our coding was written for Gatan Digital Micrograph (DM; Gatan Inc.) to quantify the dislocation density and distribution.

\section{Measurement of Dislocation Density}

There are two ways to determine dislocation density in a TEM image. The first method is 'line-intercept method' which counts the number of intersections between dislocation line and the five random lines in a TEM image (Martin et al., 1995).

$$
\rho=\frac{N}{L_{r} t}
$$

The dislocation density $\rho$ is simply the number of points $\mathrm{N}$ divided by the total length of the random lines $L_{r}$, multiplied by specimen thickness $t$.

The second method is 'line-length measurement method' which measures all dislocation lines in TEM image (Kruml et al., 2000).

$$
\rho=\frac{L_{t}}{A_{t}}
$$

In this method, the density is measured by acquiring pixel ratio on the TEM image using Forvea Pro software (reindeer graphics, USA). $\mathrm{L}_{t}$ and $\mathrm{A}$ are total length of recognized dislocation line and area of TEM image, separately. The dislocation density value is acquired by our method and the meaning of the value is discussed.

\section{Determination of Thickness of TEM Specimen}

Since the equations of dislocation density include specimen thickness, $t$, it is important to measure precisely thickness of TEM specimen. There are currently five methods are used to determine thickness of TEM specimen; analyzing thickness fringe depending on two-beam condition, contamination spot separation (CSS) method, convergent beam electron diffraction method, mean free path in electron energy loss spectroscopy and finally X-ray spectrometry method. We utilize here CSS method in scanning transmission electron microscope (STEM) mode because this is reasonably reliable and feasible method to measure the specimen thickness. Contamination carbon spots (or even longer pillars) are deposited on both sides of thin TEM specimen deliberately by prolonged exposure of intense probe in STEM mode. This method analyzes separation length of the titled pillars directly depending on the thickness of specimen.

Fig. 1A is a schematic diagram of formation of a contamination spot on the specimen. The upper side of contamination is more deposited than lower side. When the specimen is tilted by tilting angle $\gamma^{\circ}$ on the spot as illustrated in Fig. 1B, the length of separation depends on tilting angle in projected TEM images. The following equation is a relation of thickness of specimen $(\mathrm{t})$, magnification $(\mathrm{M})$ and distance of separation length $(\mathrm{r})$ in this configuration.

$$
\mathrm{t}=\frac{r}{M \sin \gamma}
$$

Determination of the thickness may be a little more complicated if the specimen itself is tilted by an angle $\varepsilon$ when the contamination is deposited. If we add the term, $\varepsilon$, thickness of specimen would be calculated by the following equation.

$$
\mathrm{t}=\frac{r \cos \varepsilon}{M \sin \gamma}
$$

\section{RESULTS AND DISCUSSION}

\section{TEM Imaging}

Fig. 2A shows dislocations in GaAs films in anti-phase boundary. Formation of annihilation due to $2^{\circ}$-off tilted $\mathrm{Si}$ during the GaAs growth is observed. Dislocation density varies and some specific regions contain less dislocation showing the higher quality of GaAs films.

\section{Image Filtering}

In order to analyze dislocation density from TEM image as shown in Fig. 2A, threshold filtering is required to distinguish dislocations from TEM image for calculations.

Fig. 2B and C are step-by-step filtered images of Fig. 2A by using 'stamp filtering' of Photoshop and 'skeletonize' filtering of Image J. The filtered image is assumed by following reason. 
A

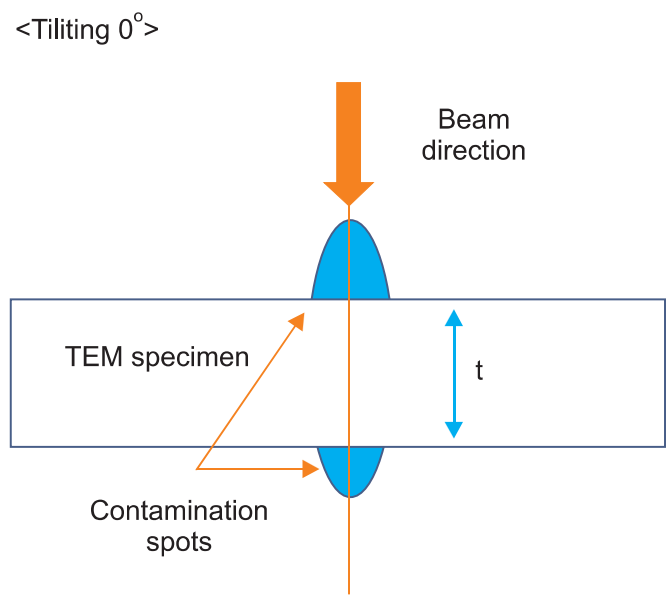

B

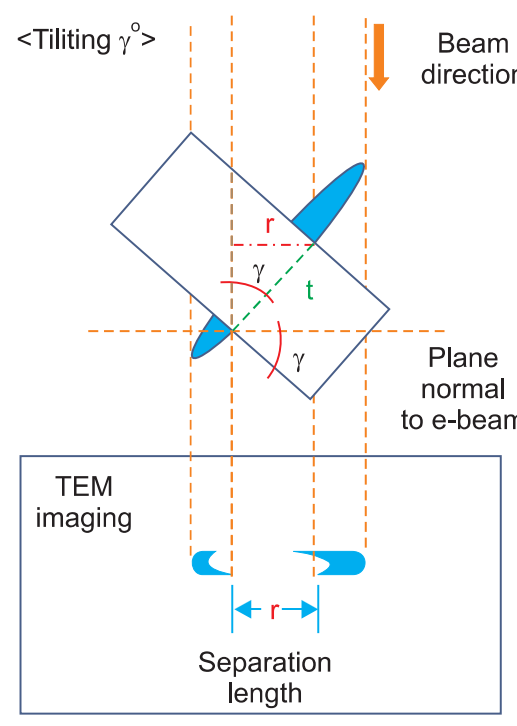

Fig. 1. Schematic of deposited contamination spot or pillars on transmission electron microscopy (TEM) specimen (A) and projected contamination spot on TEM screen at tilting $\gamma^{\circ}$ (B). t, thickness of specimen; r, distance of separation length.
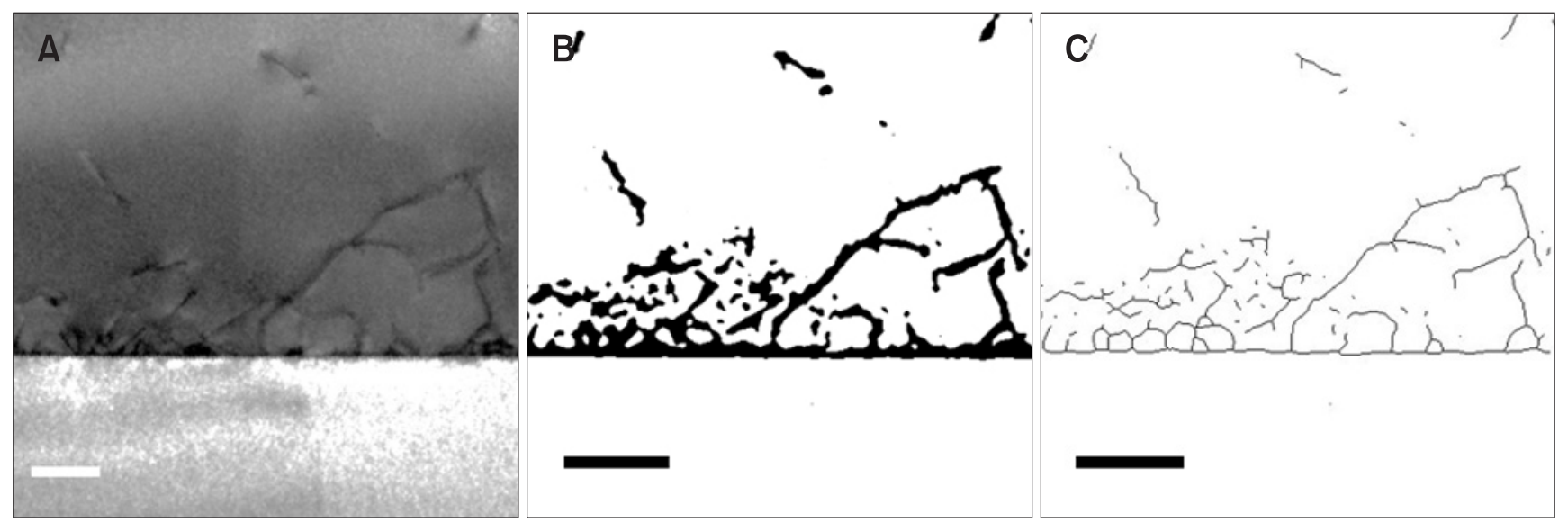

Fig. 2. Image processing steps for dislocation analysis. (A) Dislocations in transmission electron microscopy image of GaAs on Si. (B) Stamp filtered image by Photoshop ver. 8.0 (Adobe Systems Inc.). (C) Skeletonize filtered image by Image J (National Institutes of Health). Scale bars=0.2 $\mu \mathrm{m}$.

Actually, the form of the dislocations in TEM image strongly depends on the diffraction conditions and its depth in the specimen. It may appear as a single line, a double line, a wavy line or a broken line. Also, some lines may be invisible, so visible dislocations in TEM image might be a part of dislocations. Additionally, noise cannot be fully removed by the stamp filtering, so, residual noise pixels should be eliminated manually.

\section{Thickness Measurement}

Dislocation density values calculated by equation (1) and (2) are very sensitive to thickness of specimen. Measurement of accurate thickness of TEM specimen was performed using contamination pillars deposited for few minutes in STEM mode as shown in Fig. 3A. When the specimen is tilted at $20^{\circ}$ $\alpha$-tilting, the separation length of two pillars on both sides of specimen is visible in TEM imaging as shown in Fig. 3B.

In Fig. 3B, the separation length measured from tilted contamination pillars is used to calculate the thickness of TEM specimen using equation (3). Although more precise calculation may be performed using equation (4), it is hard to acquire the precise angle of between beam and specimen; $\varepsilon$. So, $\cos \varepsilon$ factor is ignored. As a result, the calculated thickness through CSS method is about $82.16 \mathrm{~nm}$.

\section{Calculation of Dislocation Density}

The dislocations are automatically detected in the image while a measuring line is scanning along the growth direction from the interface by using our own code in Gatan DM. The code is written based on equation (2). By the contrary to other methods, our method can acquire both quantitative distribution of dislocations and density information that ratio 

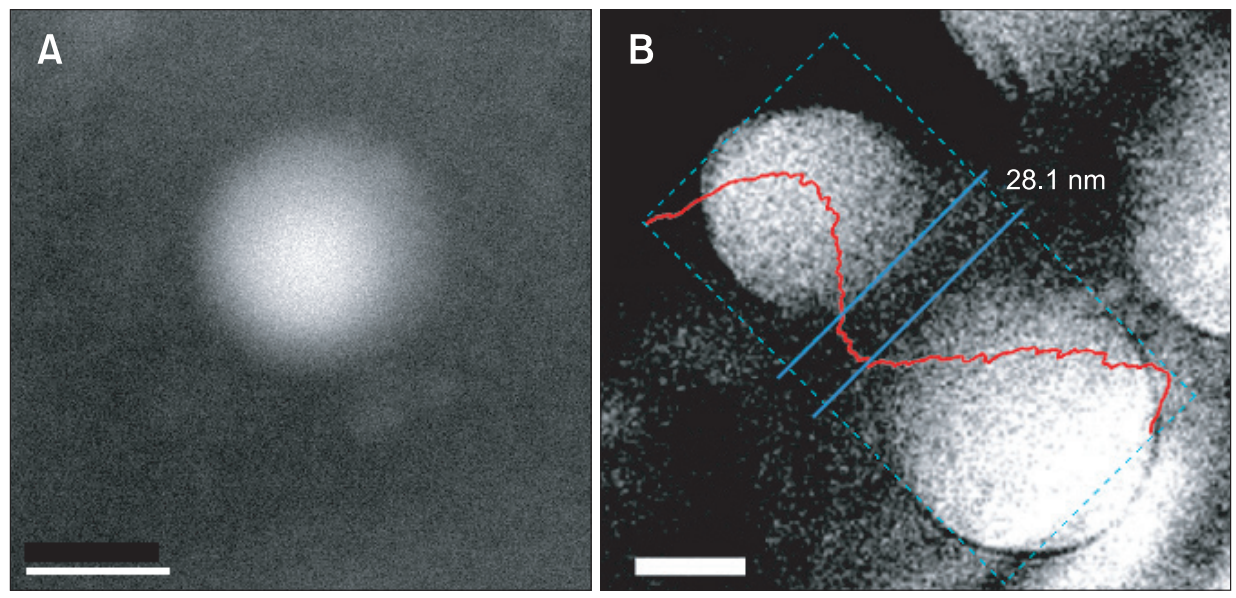

Fig. 3. (A) Transmission electron microscopy image of contamination pillars produced in scanning transmission electron microscope mode. Scale bar=50 nm. (B) Separation length of tilted contamination and red line indicated a line profile in dashed box. Separation length is determined to about $28.1 \mathrm{~nm}$. Scale bar $=500 \mathrm{~nm}$.

A

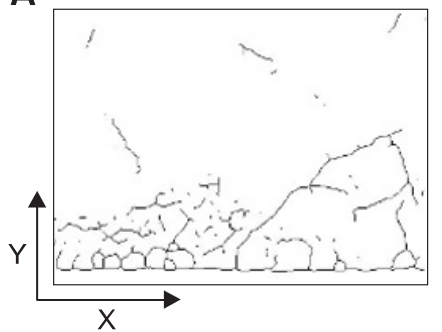

B

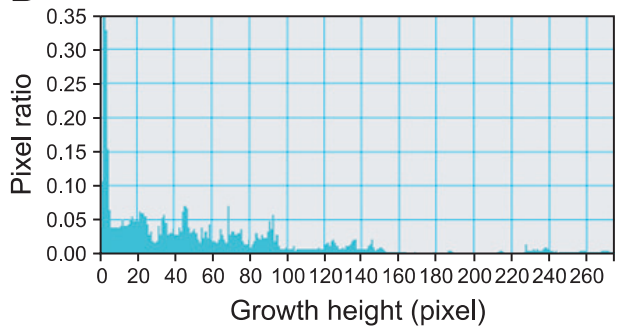

C

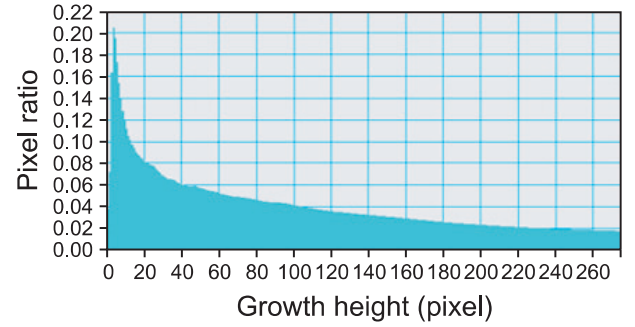

Fig. 4. (A) Filtered transmission electron microscopy image. (B) Line profile of dislocation density percent along the growth direction. (C) Accumulated dislocation density along the growth direction.

of the black pixels of dislocations to all pixels in the region of interest at the same time

Fig. 4A is filtered TEM image and Fig. 4B and $\mathrm{C}$ are line profiles of the filtered image by using our code. These line profiles show variation of quantity of dislocation line-by-line effectively by sweeping a thin line with the width of one pixel. The first profile (Fig. 4B) is the pixel ratio data of dislocation density along the growth direction and the second profile (Fig. 4C) is accumulated dislocation density along the growth direction. In other word, y value of the profile implies degree of dislocation density in region of interest. Additionally, the ratio divided by thickness of specimen indicates dislocation density. When our method applied in Fig. 4A, for instance, accumulated pixel ratio is 0.019 by sweeping line-by-line between the pixel position 0 , the interface as a reference, to 260 above. Considering calibration of pixels in the TEM image, one pixel is $3.28 \mathrm{~nm}$. Thus, calculated dislocation density results in $7.02 \times 10^{7} / \mathrm{cm}$ between the pixel position 0 to 260 , equivalent to the thickness from the interface to 852.8 $\mathrm{nm}$ in the epitaxial GaAs film. As a result using quantification method developed in this study, the dislocation density decreases with growth of epitaxial GaAs film dramatically as shown in Fig. 4C. It is note that this implies the dislocations in the epitaxial film may be reduced by self-annihilation while growth of the film.

\section{CONCLUSIONS}

For quantitative and effective evaluation of dislocation density and distribution, many tools exist. In this study, epitaxial GaAs layer on $\mathrm{Si}$ is analyzed as a model specimen. Quantification of dislocation density can be done by line-intercept method and line-length measurement method in TEM imaging. However, result of line-intercept method strongly depends on how to draw random lines and is not adequate to quantify dislocation density. Although line-length measurement pro vides more reliable data, it is difficult to quantify the dislocation density at specific growth height and observe a variation of dislocation density along the growth direction. Our coding method based on line-scanning is proposed for efficient measuring the variation of dislocation density through the film thickness. Dislocations can be detected automatically and continuously by a sweeping line in the code, and dislocation density and distribution are acquired by this method.

\section{CONFLICT OF INTEREST}

No potential conflict of interest relevant to this article was reported. 


\section{REFERENCES}

Heuser B J (1994) Small-angle neutron scattering study of dislocations in deformed single-crystal copper. J. Appl. Cryst. 28, 1020-1029.

Kawabe M and Ueda T (1987) Self-annihilation of antiphase boundary in GaAs on $\mathrm{Si}(100)$ grown by molecular beam epitaxy. Jpn. J. Appl. Phys. 24, 944-946.

Kruml T, Paidar V, and Martin J L (2000) Dislocation density in $\mathrm{Ni}_{3}(\mathrm{Al}, \mathrm{Hf}$ ). Intermetallics 8, 729 .

Martin U, Muhle U, and Heinrich O (1995) The quantitative measurement of dislocation density in the transmission electron microscope. Parkt. Metallogr. 32, 467.

Moore G E (1965) Cramming more components onto integrated circuits. Electronics 38, 114.

Wagner G (1998) Misfit strain relaxation by dislocations in InAs islands and layers epitaxially grown on (001) GaAs substrates by MOVPE. Cryst. Res. Technol. 33, 681-705. 